Infinite Dimensional Analysis, Quantum Probability and Related Topics

(2019) 1950008 (10) pages)

(C) World Scientific Publishing Company

DOI: 10.1142/S0219025719500085

\title{
A characterization of quantum Markov semigroups of weak coupling limit type
}

\author{
Franco Fagnola \\ Dipartimento di Matematica, Politecnico di Milano, \\ Piazza Leonardo da Vinci 32, I-20133 Milano, Italy \\ franco.fagnola@polimi.it \\ Roberto Quezada \\ Departamento de Matemáticas, \\ UAM-Iztapalapa, Av. San Rafael Atlixco 186, \\ Col. Vicentina, 09340 México D.F., México \\ roqb@xanum.uam.mx \\ Received 21 March 2017 \\ Accepted 6 May 2019 \\ Published \\ Communicated by A. Chebotarev
}

\begin{abstract}
We characterize generators of quantum Markov semigroups leaving invariant a maximal abelian purely atomic algebra and certain operator subspaces associated with it in a natural way. From this result, we also establish a characterization of generators of quantum Markov semigroups of weak coupling limit type associated with a nondegenerate Hamiltonian.

Keywords: Quantum Markov semigroup; weak coupling limit-type generator; invariant operator subspace.
\end{abstract}

AMS Subject Classification: 47D07, 82C10, 82C31

\section{Introduction}

Weak coupling limit-type (WCLT) quantum Markov semigroups (see Ref. 2) are semigroups of completely positive maps, closely related with a discrete spectrum Hamiltonian $H_{S}$ with remarkable structural properties. Their invariant states satisfy the local Kubo-Martin-Schwinger (KMS) condition, see Ref. 2 that distinguishes, among the states of the dynamics (i.e. functions of the invariants of motion in the commutant of the Hamiltonian $\left.\left\{H_{S}\right\}^{\prime}\right)$, those which are functions of the Hamiltonian, i.e. in the von Neumann algebra $\rho \in\left\{H_{S}\right\}^{\prime \prime}$, the double commutant of $H_{S}$. Generators of these semigroups are written as the sum of other generators, one for each Bohr frequency, with completely positive part with multiplicity one (in 

at the first instance.

the zero temperature case) or two (in the positive temperature case). Their structure is simple enough to allow explicit computation of their stationary states, but rich enough to exhibit detailed balance (equilibrium) as well as nondetailed balance (but local equilibrium) invariant states. Moreover, WCLT generators leave invariant, not only the commutant of the system Hamiltonian, but also a multiplicity of operator subspaces of $\mathcal{B}(\mathrm{h})$. In Ref. 2 , we conjectured that this property characterizes WCLT generators. This paper is aimed at investigating this conjecture for QMSs with nondegenerate Hamiltonian $H_{S}$.

With this motivation, as a first step, we characterize QMSs leaving invariant the maximal abelian purely atomic algebra $\mathcal{D}_{0}$ generated by the system Hamiltonian $H_{S}$ and the operator subspaces $\mathcal{D}_{n}$ (2.7) (Property $\mathbf{P}$ in Sec. 2) associated with it in a natural way. Theorem 3.1 shows that one can find a Gorini-KossakowskiSudharshan-Lindblad (GKSL) representation of their generators with all operators $L_{\ell}$ in the completely positive part of the generator belonging to some $\mathcal{D}_{n}$ and all the other operators in the maximal (diagonal) algebra $\mathcal{D}_{0}$.

This shows that the conjecture as stated in Ref. 2, in general, is not true. As a matter of fact, one could consider generators with all operators in a GKSL representation belonging to $\mathcal{D}_{0}$ which are not of WCLT but leave invariant all the operator spaces $\mathcal{D}_{n}$ for all $n$. However, if we further detail a bit the properties of the operators in the GKSL representation as in Theorem 4.1, we can prove the conjecture with a slightly different formulation.

We would like to emphasize here that Property $\mathbf{P}$ is very useful in the study of several QMSs because, roughly speaking, it allows one to reduce the dimension of the space where the semigroup acts, slicing up it into its subspaces $\mathcal{D}_{n}$. This happens, for instance, in the study of the spectral gap (see Refs. 5] and 7) and the entropy production rate (see Ref. 6).

QMSs leaving invariant a maximal abelian algebra have been studied in Ref. 4. This property, however, is much weaker than Property $\mathbf{P}$ considered here and does not allow to draw conclusions on the shape of the operators in a GKSL representation of the generator.

\section{Semigroups of WCLT}

Let $H_{S}$ be a positive self-adjoint operator (reference Hamiltonian) acting on a separable complex Hilbert space $\mathrm{h}$ with discrete spectral decomposition

$$
H_{S}=\sum_{\varepsilon_{m} \in \operatorname{Sp}(H)} \varepsilon_{m} P_{\varepsilon_{m}},
$$

where $\varepsilon_{m}$, with $\varepsilon_{m}<\varepsilon_{n}$ for $m<n$, are the eigenvalues of $H_{S}$ and $P_{\varepsilon_{m}}$ are the corresponding eigenspaces. We consider WCLT-bounded generators of QMSs, associated with the Hamiltonian $H_{S}$, of the form

$$
\mathcal{L}=\sum_{\omega \in B_{+}} \mathcal{L}_{\omega},
$$




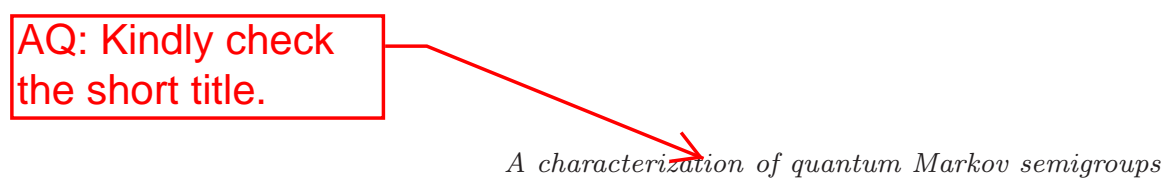

where $B_{+}$is the set of all Bohr frequencies (Arveson spectrum)

$$
B_{+}:=\left\{\left(\varepsilon_{n}, \varepsilon_{m}\right): \varepsilon_{n}-\varepsilon_{m}>0\right\} .
$$

For every Bohr frequency $\omega, \mathcal{L}_{\omega}$ is a generator with the GKSL structure

$$
\begin{gathered}
\mathcal{L}_{\omega}(x)=\mathrm{i}\left[H_{\omega}, x\right]-\frac{\Gamma_{-\omega}}{2}\left(D_{\omega}^{*} D_{\omega} x-2 D_{\omega}^{*} x D_{\omega}+x D_{\omega} D_{\omega}^{*}\right) \\
-\frac{\Gamma_{+\omega}}{2}\left(D_{\omega} D_{\omega}^{*} x-2 D_{\omega} x D_{\omega}^{*}+x D_{\omega} D_{\omega}^{*}\right)
\end{gathered}
$$

for all $x \in \mathcal{B}(\mathrm{h})$, with Kraus operators $D_{\omega}$ defined by

$$
D_{\omega}=\sum_{\left(\varepsilon_{n}, \varepsilon_{m}\right) \in B_{+, \omega}} P_{\varepsilon_{m}} D P_{\varepsilon_{n}},
$$

where $B_{+, \omega}=\left\{\left(\varepsilon_{n}, \varepsilon_{m}\right): \varepsilon_{n}-\varepsilon_{m}=\omega\right\}, D$ belongs to $\mathcal{B}(\mathrm{h})$, the von Neumann algebra of all bounded operators on $\mathrm{h}, \Gamma_{-\omega}, \Gamma_{+\omega}$ are nonnegative real constants with $\Gamma_{-\omega}+\Gamma_{+\omega}>0$ and $H_{\omega}$ is a bounded self-adjoint operator on $\mathrm{h}$ commuting with $H_{S}$.

In the case when the set of Bohr frequencies is infinite, for $\mathcal{L}$ to be the generator of a norm continuous QMS, the series

$$
\sum_{\omega \in B_{+}}\left(\Gamma_{-\omega} D_{\omega}^{*} D_{\omega}+\Gamma_{+\omega} D_{\omega} D_{\omega}^{*}\right)
$$

must be strongly convergent in $\mathcal{B}(\mathrm{h})$, see Corollary 30.13 on p. 268 and Theorem 30.16 on p. 271 of Ref. 10.

The class of WCLT generators leaves invariant the commutant $\left\{H_{S}\right\}^{\prime}$ of the Hamiltonian as well as several subspaces of off-diagonal operators, see Corollary 3.2 in Ref. 2 where it was conjectured that this property characterizes the WCLT generators.

In this paper, we suppose that the Hamiltonian $H_{S}$ is also nondegenerate, namely, in the spectral representation (2.1), spectral projections $P_{\epsilon_{m}}$ are onedimensional.

In order to introduce our framework, we denote by $\left(e_{m}\right)_{m \geq 0}$ an orthonormal basis of $\mathrm{h}$ of eigenvectors of $H_{S}$, i.e. $H_{S} e_{m}=\varepsilon_{m} e_{m}$ for all $m \geq 0$. Consider the operator subspaces $\mathcal{D}_{n}$ with $n \in \mathbb{Z}$ defined by

$$
\mathcal{D}_{n}=\left\{\sum_{i \geq \max (0,-n)} z_{i}\left|e_{i}\right\rangle\left\langle e_{i+n}|| z_{i} \in \mathbb{C}, \sup _{i \geq \max (0,-n)}\left|z_{i}\right|<\infty\right\} .\right.
$$

Clearly, $\mathcal{D}_{0}$ is the maximal abelian von Neumann subalgebra of $\mathcal{B}(\mathrm{h})$ generated by one-dimensional projections $\left|e_{i}\right\rangle\left\langle e_{i}\right|$.

Under the above assumptions, WCLT generators enjoy the following.

Property P. For every $n \in \mathbb{Z}$ and for every Bohr frequency $\omega$, the operator subspace $\mathcal{D}_{n}$ is invariant under the action of $\mathcal{L}_{\omega}$. 


\section{F. Fagnola \& R. Quezada}

Proof. Indeed, if $z \in \mathcal{D}_{n}$, denoting by $e_{i}^{ \pm \omega}$ the eigenvector of the eigenvalue $\varepsilon_{i} \pm \omega$, an easy computation yields

$$
\begin{aligned}
& D_{\omega} z D_{\omega}^{*}=\sum_{i \geq \max (0,-n)} z_{i}\left\langle e_{i}, D e_{i}^{+\omega}\right\rangle\left\langle e_{i+n}^{+\omega}, D^{*} e_{i+n}\right\rangle\left|e_{i}\right\rangle\left\langle e_{i+n}\right|, \\
& D_{\omega}^{*} z D_{\omega}=\sum_{i \geq \max (0,-n)} z_{i}\left\langle e_{i}, D^{*} e_{i}^{-\omega}\right\rangle\left\langle e_{i+n}^{-\omega}, D e_{i+n}\right\rangle\left|e_{i}\right\rangle\left\langle e_{i+n}\right|, \\
& D_{\omega}^{*} D_{\omega} z=\sum_{i \geq \max (0,-n)} z_{i}\left\langle e_{i}, D e_{i}^{+\omega}\right\rangle\left\langle e_{i}^{+\omega}, D^{*} e_{i}\right\rangle\left|e_{i}\right\rangle\left\langle e_{i+n}\right|, \\
& D_{\omega} D_{\omega}^{*} z=\sum_{i \geq \max (0,-n)} z_{i}\left\langle e_{i}, D^{*} e_{i}^{-\omega}\right\rangle\left\langle e_{i}^{-\omega}, D e_{i}\right\rangle\left|e_{i}\right\rangle\left\langle e_{i+n}\right|
\end{aligned}
$$

and, taking the adjoint, similar formulae hold for $z D_{\omega}^{*} D_{\omega}, z D_{\omega} D_{\omega}^{*}$. As a consequence, all the above operators belong to $\mathcal{D}_{n}$ for all $\omega$.

The family of subspaces $\mathcal{D}_{n}$ has a rich structure. One can easily verify that each $\mathcal{D}_{n}$ is a pre-Hilbert $\mathcal{D}_{0}$-module with the inner product defined for $z, w \in \mathcal{D}_{n}$ as

$$
\langle z, w\rangle=z^{*} w \in \mathcal{D}_{0} .
$$

Moreover we have the following.

Lemma 2.1. (i) Every element $X \in \mathcal{B}(\mathrm{h})$ can be represented as $X=\sum_{n \in \mathbb{Z}} X_{n}$ with $X_{n} \in \mathcal{D}_{n}$, the series being strongly convergent,

(ii) If $W=\sum_{m \in \mathbb{Z}} W_{m}$ and $V=\sum_{m \in \mathbb{Z}} V_{m}$ are two bounded operators, then

$$
W_{m}^{*} \mathcal{D}_{n} V_{m^{\prime}} \subset \mathcal{D}_{n}
$$

if and only if $m=m^{\prime}$.

Proof. (i) It suffices to note that $\mathbb{1}=\sum_{m \geq 0} P_{\varepsilon_{m}}$ and the series is strongly convergent. Since the product $\left(A_{n} B_{n}\right)_{n}$ of two strongly convergent sequences $\left(A_{n}\right)_{n}$, $\left(B_{n}\right)_{n}$ in $\mathcal{B}(\mathrm{h})$ is a strongly convergent sequence because, for all $u \in \mathrm{h}$,

$$
\left\|A_{n} B_{n} u-A B u\right\| \leq\left\|A_{n}\left(B_{n}-B\right) u\right\|+\left\|\left(A_{n}-A\right) B u\right\|,
$$

and $\left(B_{n}\right)_{n}$ is uniformly bounded in norm by the uniform boundedness principle, we have

$$
X=\sum_{m, m^{\prime}} P_{\varepsilon_{m^{\prime}}} X P_{\varepsilon_{m}}=\sum_{\omega} \sum_{\left\{\left(\varepsilon_{m^{\prime}}, \varepsilon_{m}\right) \mid \varepsilon_{m}-\varepsilon_{m^{\prime}}=\omega\right\}} P_{\varepsilon_{m^{\prime}}} X P_{\varepsilon_{m}},
$$

where the sum on $\omega$ is on all differences $\varepsilon_{m}-\varepsilon_{m^{\prime}}$ of eigenvalues of $H_{S}$ (not only strictly positive Bohr frequencies). 
(ii) Now, for every $k, k^{\prime}$, we have that

$$
\begin{aligned}
W_{m}^{*}\left|e_{k}\right\rangle\left\langle e_{k^{\prime}}\right| V_{m^{\prime}} & =\sum_{j, j^{\prime} \geq 0}\left\langle W e_{j+m}, e_{j}\right\rangle\left\langle e_{j^{\prime}}, V e_{j^{\prime}+m^{\prime}}\right\rangle \delta_{j, k} \delta_{j^{\prime}, k^{\prime}}\left|e_{j+m}\right\rangle\left\langle e_{j^{\prime}+m^{\prime}}\right| \\
& =\left\langle W e_{k+m}, e_{k}\right\rangle\left\langle V e_{k^{\prime}+m^{\prime}}, e_{k^{\prime}}\right\rangle\left|e_{k+m}\right\rangle\left\langle e_{k^{\prime}+m^{\prime}}\right| \in \mathcal{D}_{k^{\prime}-k}
\end{aligned}
$$

if and only if $m=m^{\prime}$. This proves the lemma.

\section{Characterization of QMSs Leaving all $\mathcal{D}_{n} \mathrm{~s}$ Invariant}

In this section, we prove that invariance of the operator spaces $\mathcal{D}_{n}$ for the generator $\mathcal{L}$ implies that it can be decomposed as the sum of other generators, each one of them with completely positive part with multiplicity one and leaving all operator spaces $\mathcal{D}_{n}$ invariant with a special GKSL representation. More precisely, we prove the following.

Theorem 3.1. Let $\mathcal{L}$ be the generator of a norm continuous $Q M S$ on $\mathcal{B}(\mathrm{h})$ such that $\mathcal{L}\left(\mathcal{D}_{n}\right) \subseteq \mathcal{D}_{n}$ for all $n$ and operator spaces $\mathcal{D}_{n}$ as above determined by a given orthonormal basis $\left(e_{n}\right)_{n \geq 0}$. Then there exists a GKSL representation of the generator $\mathcal{L}$

$$
\mathcal{L}(x)=\mathrm{i}[H, x]-\frac{1}{2} \sum_{\ell \geq 1}\left(L_{\ell}^{*} L_{\ell} x-2 L_{\ell}^{*} x L_{\ell}+x L_{\ell}^{*} L_{\ell}\right)
$$

with $L_{\ell} \in \mathcal{D}_{n_{\ell}}$ for all $\ell$ and some $n_{\ell}$, the series $\sum_{\ell \geq 1} L_{\ell}^{*} L_{\ell}$ strongly convergent and $H=H^{*} \in \mathcal{D}_{0}$.

The first step in the proof is the following.

Lemma 3.1. Under the assumptions of Theorem 3.1, there exists a decomposition

$$
\mathcal{L}(x)=G^{*} x+\Phi(x)+x G
$$

with $G \in \mathcal{D}_{0}$ and $\Phi$ a completely positive map on $\mathcal{B}(\mathrm{h})$ such that $\Phi_{\omega}(\mathbb{1})=-G-G^{*}$ and $\Phi\left(\mathcal{D}_{n}\right) \subseteq \mathcal{D}_{n}$ for all $n \in \mathbb{Z}$.

Proof. It suffices to recall (see, e.g., Theorem 3.14 and Eq. (3.11) of Ref. 9) that we can find a GKSL decomposition of the generator by fixing a unit vector $e$ and taking as operator $G$ the adjoint of the operator $G^{*}$ defined by

$$
G^{*} u=\mathcal{L}(|u\rangle\langle e|) e-\frac{1}{2}\langle e, \mathcal{L}(|e\rangle\langle e|) e\rangle u
$$

for all $u \in \mathrm{h}$. Therefore, if we choose $e=e_{0}$, then, putting $2 c_{0}=\left\langle e_{0}, \mathcal{L}\left(\left|e_{0}\right\rangle\left\langle e_{0}\right|\right) e_{0}\right\rangle$,

$$
\begin{aligned}
G^{*} e_{i} & =\mathcal{L}\left(\left|e_{i}\right\rangle\left\langle e_{0}\right|\right) e_{0}-c_{0} e_{i} \\
& =\sum_{j \geq 0} z_{i j}\left|e_{i+j}\right\rangle\left\langle e_{j}\right| e_{0}-c_{0} e_{i} \\
& =\left(z_{i i}-c_{0}\right) e_{i}
\end{aligned}
$$

for all $i$. In other words, each vector $e_{i}$ is an eigenvector for $G$. 


\section{F. Fagnola \& R. Quezada}

Now, we consider the completely positive part of the generator.

Theorem 3.2. Let $\Phi$ be a completely positive map on $\mathcal{B}(\mathrm{h})$ such that $\Phi\left(\mathcal{D}_{n}\right) \subseteq \mathcal{D}_{n}$ for all $n$. Then there exists a Kraus representation $\Phi(x)=\sum_{\ell \geq 1} L_{\ell}^{*} x L_{\ell}$ in which each $L_{\ell}$ belongs to some $\mathcal{D}_{m}$.

Proof. Let $\Phi(x)=\sum_{\ell \geq 1} V_{\ell} x V_{\ell}^{*}$ be a minimal (i.e. with the minimum number of operators $V_{\ell}$ ) Kraus representation of $\Phi$ with operators $V_{\ell} \in \mathcal{B}(\mathrm{h})$ such that the series $\sum_{\ell \geq 1} V_{\ell} V_{\ell}^{*}=\Phi(\mathbb{1})$ is strongly convergent.

For all $j, k$, define $v_{\ell}(j, k)=\left\langle e_{j}, V_{\ell} e_{k}\right\rangle$. Collections of complex numbers $v(j, k)=$ $\left(v_{\ell}(j, k)\right)_{\ell \geq 1}$ can be viewed as vectors in the multiplicity space $\mathbf{k}$ of the Kraus representation of $\Phi$, indeed,

$$
\|v(j, k)\|^{2}=\sum_{\ell \geq 1}\left|v_{\ell}(j, k)\right|^{2}=\sum_{\ell \geq 1}\left\langle e_{k}, V_{\ell}^{*} e_{j}\right\rangle\left\langle V_{\ell}^{*} e_{j}, e_{k}\right\rangle=\left\langle e_{k}, \Phi\left(\left|e_{j}\right\rangle\left\langle e_{j}\right|\right) e_{k}\right\rangle<\infty .
$$

Writing $V_{\ell} e_{i}=\sum_{k} v_{\ell}(k, i) e_{k}$, a straightforward computation yields

$$
\Phi\left(\left|e_{i}\right\rangle\left\langle e_{j}\right|\right)=\sum_{\ell, k, m} v_{\ell}(k, i) \overline{v_{\ell}(m, j)}\left|e_{k}\right\rangle\left\langle e_{m}\right|,
$$

so that $\Phi$-invariance of $\mathcal{D}_{n}$ implies

$$
\begin{aligned}
& \langle v(k, i), v(m, j)\rangle_{\mathrm{k}}=\sum_{\ell} v_{\ell}(k, i) \overline{v_{\ell}(m, j)}=0, \\
& \text { whenever } j-i \neq m-k, \quad \text { i.e. } j-m \neq i-k .
\end{aligned}
$$

In other words, vectors $v(k, i), v(m, j)$ in $\mathrm{k}$ are orthogonal if $j-m \neq i-k$.

It follows that one can find a new basis of $\mathrm{k}$ and a family of disjoint (possibly infinite) subsets $I(k-i)$ of the set of indices (each difference is associated with one and only one subset!) such that, denoting by $U$ the unitary operator of the change of basis, the following property holds:

$$
\begin{aligned}
& \text { for each } \ell \text { and differences } k^{\prime}-i^{\prime} \neq k-i, \\
& \text { either }(U v(k, i))_{\ell}=0 \quad \text { or } \quad\left(U v\left(k^{\prime}, i^{\prime}\right)\right)_{\ell}=0 .
\end{aligned}
$$

Clearly, coordinates of vectors $v(k, i)$ in the new basis are given by

$$
(U v(k, i))_{\ell}=\sum_{\ell^{\prime}} U_{\ell \ell^{\prime}} v_{\ell^{\prime}}(k, i) .
$$

$$
L_{\ell}^{*}=\sum_{k^{\prime}, i^{\prime}}\left(U v\left(k^{\prime}, i^{\prime}\right)\right)_{\ell}\left|e_{k^{\prime}}\right\rangle\left\langle e_{i^{\prime}}\right| .
$$


Since $L_{\ell}^{*}=\sum_{\ell^{\prime}} U_{\ell \ell^{\prime}} V_{\ell^{\prime}}$, the operator $\sum_{\ell} L_{\ell}^{*} x L_{\ell}$ is given by

$$
\begin{aligned}
& \sum_{k^{\prime}, i^{\prime}, k^{\prime \prime}, i^{\prime \prime}, \ell^{\prime}, m^{\prime}, \ell} U_{\ell \ell^{\prime}} v_{\ell^{\prime}}\left(k^{\prime}, i^{\prime}\right) \overline{U_{\ell m^{\prime}} v_{m^{\prime}}\left(k^{\prime \prime}, i^{\prime \prime}\right)}\left|e_{k^{\prime}}\right\rangle\left\langle e_{i^{\prime}}|x| e_{i^{\prime \prime}}\right\rangle\left\langle e_{k^{\prime \prime}}\right| \\
& =\sum_{k^{\prime}, i^{\prime}, k^{\prime \prime}, i^{\prime \prime}, \ell^{\prime}, m^{\prime}}\left(\sum_{\ell} U_{\ell \ell^{\prime}} \bar{U}_{\ell m^{\prime}}\right) v_{\ell^{\prime}}\left(k^{\prime}, i^{\prime}\right) \overline{v_{m^{\prime}}\left(k^{\prime \prime}, i^{\prime \prime}\right)}\left|e_{k^{\prime}}\right\rangle\left\langle e_{i^{\prime}}|x| e_{i^{\prime \prime}}\right\rangle\left\langle e_{k^{\prime \prime}}\right| \\
& =\sum_{\ell^{\prime}, k^{\prime}, i^{\prime}, k^{\prime \prime}, i^{\prime \prime}} v_{\ell^{\prime}}\left(k^{\prime}, i^{\prime}\right) \overline{v_{\ell^{\prime}}\left(k^{\prime \prime}, i^{\prime \prime}\right)}\left|e_{k^{\prime}}\right\rangle\left\langle e_{i^{\prime}}|x| e_{i^{\prime \prime}}\right\rangle\left\langle e_{k^{\prime \prime}}\right| \\
& =\sum_{\ell^{\prime}, i^{\prime}, i^{\prime \prime}}\left|V_{\ell^{\prime}} e_{i^{\prime}}\right\rangle\left\langle e_{i^{\prime}}|x| e_{i^{\prime \prime}}\right\rangle\left\langle V_{\ell^{\prime}} e_{i^{\prime \prime}}\right| \\
& =\sum_{\ell, i^{\prime}, i^{\prime \prime}} V_{\ell}\left|e_{i^{\prime}}\right\rangle\left\langle e_{i^{\prime}}|x| e_{i^{\prime \prime}}\right\rangle\left\langle e_{i^{\prime \prime}}\right| V_{\ell}^{*} \\
& =\sum_{\ell} V_{\ell} x V_{\ell}^{*} .
\end{aligned}
$$

Moreover, $L_{\ell}$ belongs to some $\mathcal{D}_{m}$ because, by (3.4), there is one and only one difference $m=k^{\prime}-i^{\prime}$ (but possibly infinitely many pairs $\left(i^{\prime}, k^{\prime}\right)$ with $k^{\prime}-i^{\prime}=m$ ) for which $\left(U v\left(k^{\prime}, i^{\prime}\right)\right)_{\ell}$ may be nonzero.

We denote by $S$ the right shift operator $S e_{n}=e_{n+1}$. The following corollary immediately follows.

Corollary 3.1. Let $\Phi$ be a completely positive map on $\mathcal{B}(\mathrm{h})$ such that $\Phi\left(\mathcal{D}_{n}\right) \subseteq \mathcal{D}_{n}$ for all $n$. Then there exists a Kraus representation $\Phi(x)=\sum_{\ell \geq 1} L_{\ell}^{*} x L_{\ell}$ in which each $L_{\ell}$ can be written in one of the following forms:

$$
S^{n} M \quad \text { or } \quad S^{* n} M
$$

for some $n \geq 0$ and some multiplication operator $M$.

Proof. Clear from the definition of $\mathcal{D}_{n}$. Indeed, if $Z=\sum_{i \geq \max (0,-n)} z_{i}\left|e_{i}\right\rangle\left\langle e_{i+n}\right|$ and $n \geq 0$, say, so that $Z=\sum_{j \geq 0} z_{j}\left|e_{j}\right\rangle\left\langle e_{j+n}\right|$, considering the multiplication operator $M=\sum_{j \geq 0} z_{j}\left|e_{j+n}\right\rangle\left\langle e_{j+n}\right|$, we have $Z=S^{* n} M$. In a similar way, if $n<$ $0, Z=\sum_{j \geq 0} z_{j-n}\left|e_{j-n}\right\rangle\left\langle e_{j}\right|$ and so, defining $M=\sum_{j \geq 0} z_{j-n}\left|e_{j}\right\rangle\left\langle e_{j}\right|$, we have $Z=S^{-n} M$.

Proof of Theorem 3.1. Consider a representation of $\mathcal{L}$ as in (3.2), Lemma 3.1, and a Kraus representation of the completely positive map $\Phi$ as in Theorem 3.2 with all $L_{\ell}$ in some $\mathcal{D}_{n}$. Since $G \in \mathcal{D}_{0}$, we have also $G^{*} \in \mathcal{D}_{0}$ so that its anti-self-adjoint part $H=\left(G^{*}-G\right) /(2 \mathrm{i})$ belongs to $\mathcal{D}_{0}$. 


\section{Characterization of QMSs of WCLT}

The following result gives our characterization of QMSs of WCLT.

Theorem 4.1. Let $\mathcal{L}$ be the generator of a norm continuous $Q M S$ on $\mathcal{B}(\mathrm{h})$ such that $\mathcal{L}\left(\mathcal{D}_{n}\right) \subseteq \mathcal{D}_{n}$ for all $n$ and operator spaces $\mathcal{D}_{n}$ as above determined by a given orthonormal basis $\left(e_{n}\right)_{n \geq 0}$ and consider a GKSL representation (3.1) by means of operators $H=H^{*} \in \mathcal{D}_{0}$ and $L_{\ell}=S^{* n_{\ell}} M_{\ell}$ for $n_{\ell} \geq 0, L_{\ell}=S^{-n_{\ell}} M_{\ell}$ for $n_{\ell}<0$. $\mathcal{L}$ is a generator of WCLT if and only if

(1) $n_{\ell} \neq 0$ for all $\ell \geq 1$.

(2) The function $\ell \mapsto n_{\ell}$ is injective.

(3) For all pair $(\ell, l)$ such that $n_{\ell}=-n_{l}$, there exist complex constants $z_{\ell}, w_{l}$ such that $z_{\ell} M_{\ell}=w_{l} \bar{M}_{l}$ (i.e. $z_{\ell} M_{\ell}=w_{l} M_{l}^{*}$ since $M_{\ell}$ and $M_{l}$ are diagonal).

Proof. Generators of WCLT clearly enjoy the properties (1)-(3). Conversely, if these properties hold, let $K=\sum_{m \geq 0} m\left|e_{m}\right\rangle\left\langle e_{m}\right|$ and let

$$
\begin{aligned}
& \Lambda^{-}=\left\{\ell \geq 1: L_{\ell}=S^{*\left(-n_{\ell}\right)} M_{\ell} \text { with } n_{\ell}<0\right\}, \\
& \Lambda^{+}=\left\{\ell \geq 1: L_{\ell}=S^{n_{\ell}} M_{\ell} \text { with } n_{\ell}>0 \text { and } \nexists k \text { s.t. } L_{k}=S^{*\left(n_{\ell}\right)} M_{k}\right\}
\end{aligned}
$$

(recall the convention $S^{* m}=S^{-m}$ for $m<0$ ). In other words, $\Lambda^{-}$is the set of indices corresponding to operators $L_{\ell}$ which are of annihilation type, mapping each level $j$ into the lower level $j+n_{\ell}, \Lambda^{+}$is the set of indices corresponding to operators $L_{\ell}$ of creation type, mapping each lower level $j$ into the upper level $j+n_{\ell}$, for which there exists no another associated operator $L_{k}$ of annihilation type mapping the same upper levels $j+n_{\ell}$ into the same lower levels $j$.

The sets $\Lambda^{-}$and $\Lambda^{+}$form a partition of the set of indices $\ell$. Define

$$
D=\sum_{\ell \in \Lambda^{-}} S^{*\left(-n_{\ell}\right)} M_{\ell}+\sum_{\ell \in \Lambda^{+}} S^{* n_{\ell}} M_{\ell}
$$

Clearly,

$$
\sum_{\left\{\left(m, m^{\prime}\right): m-m^{\prime}=\left|n_{\ell}\right|\right\}} P_{m^{\prime}} D P_{m}= \begin{cases}S^{*\left(-n_{\ell}\right)} M_{\ell} & \text { if } n_{\ell}<0, \\ S^{* n_{\ell}} M_{\ell}^{*} & \text { if } n_{\ell}>0 .\end{cases}
$$

Recalling that, for all $\ell \in \Lambda^{-}, z_{\ell} M_{\ell}=w_{l} M_{l}^{*}$ for another index $l$ such that $n_{\ell}=-n_{l}$, with $z_{\ell}=0$ if and only if there is no creation type operator associated with $L_{\ell}$, it follows that the generator $\mathcal{L}$ is of WCLT with $B_{+}=\left\{\left|n_{\ell}\right|: \ell \geq 1\right\}$ and

- for $\ell \in \Lambda^{-}, L_{\ell}=S^{*\left(-n_{\ell}\right)} M_{\ell}, \Gamma_{-\left|n_{\ell}\right|}=1, \Gamma_{+\left|n_{\ell}\right|}=\overline{w_{\ell} z_{\ell}^{-1}}$ if there is an associated creation type operator, $\Gamma_{+\left|n_{\ell}\right|}=0$ if not,

- for $\ell \in \Lambda^{+}, L_{\ell}=S^{* n_{\ell}} M_{\ell}^{*}, \Gamma_{+n_{\ell}}=1, \Gamma_{-n_{\ell}}=0$.

This completes the proof. 
Remark 4.1. It is worth noticing here that a system with a generic Hamiltonian $H_{S}$, weakly coupled with a reservoir with an interaction like $D \otimes A(g)+D^{*} \otimes A^{*}(g)$, gives rise to a generic QMS (see Refs. 1 and 8 and the references therein). However, a highly degenerate system Hamiltonian such as the number operator on the onemode Fock space $\Gamma(\mathbb{C}) \simeq \ell^{2}(\mathbb{N})$ with a suitable interaction operator $D$ may give rise to a generic QMS as well. Indeed, if we consider the canonical orthonormal basis $\left(e_{n}\right)_{n \geq 0}$, the system Hamiltonian $N$ and interaction operator $D$

$$
N=\sum_{n \geq 0} n\left|e_{n}\right\rangle\left\langle e_{n}\left|, \quad D=\sum_{k \geq 1}\right| e_{2^{k-1}}\right\rangle\left\langle e_{2^{k}}\right|,
$$

then one immediately sees that the only nonzero $D_{\omega} \mathrm{s}$ (see (2.5)) are those corresponding to frequencies $\omega=2^{k}-2^{k-1}=2^{k-1}$. Choosing constants $\Gamma_{-\omega}>\Gamma_{+\omega}>0$ in such a way that the series (2.6) is strongly convergent, we find a generic QMS. Indeed, this QMS could also arise from the weak coupling limit of the system Hamiltonian

$$
H_{S}=\sum_{k \geq 1} 2^{k}\left|e_{k}\right\rangle\left\langle e_{k}\right|
$$

and $2^{k}-2^{j}=2^{k^{\prime}}-2^{j^{\prime}}$ if and only if $k=k^{\prime}$ and $j=j^{\prime}$. This can be seen supposing that $k \geq k^{\prime}$ (if not exchange $k$ and $k^{\prime}$ ) and $k>j$ (if not exchange $k$ and $j$ ) to fix the ideas. In this case, the identity $2^{k}-2^{j}=2^{k^{\prime}}-2^{j^{\prime}}$ with $k=k^{\prime}$ implies $j=j^{\prime}$. Moreover, it cannot hold for $k>k^{\prime}$ because it is equivalent to $2^{k-k^{\prime}}-2^{j-k^{\prime}}=$ $1-2^{j^{\prime}-k^{\prime}}$ and one can see that $2^{k-k^{\prime}}-2^{j-k^{\prime}}>1>1-2^{j^{\prime}-k^{\prime}}$.

Remark 4.2. The class of WCLT generators introduced in Ref. 2 correspond to the case when the interaction is of multiplicity one. More general interactions are possible, like those of dipole type $\sum_{j}\left(D_{j}^{*} \otimes A\left(g_{j}\right)+D_{j} \otimes A^{*}\left(g_{j}\right)\right)$, studied in Ref. [3, where $D_{j}$ are operators acting on $\mathrm{h}$ and $A\left(g_{j}\right), A^{*}\left(g_{j}\right)$ are annihilation and creation operators of a quantum field. WCLT generators with interaction of multiplicity greater than one will be considered in the nearest future.

\subsection{Circulant generators are not WCLT}

Circulant generators are another class of finite-dimensional generators simple enough to allow explicit computation of their invariant states but rich enough to go beyond detailed balance, see Ref. 6. They leave invariant operator subspaces $\left(\mathcal{B}_{n}\right)_{0 \leq n \leq d-1}$ similar to our subspaces $\left(\mathcal{D}_{n}\right)_{-d \leq n \leq d}$ but with a cyclic (or circulant) structure. Due to this fact, they are not generic WCLT with a nondegenerate Hamiltonian.

\section{Acknowledgments}

The financial support from CONACYT-Mexico (Grant 221873) and PRODEP Red de Análisis Italia-UAM México is gratefully acknowledged. 


\section{References}

1. L. Accardi, F. Fagnola and S. Hachicha, Generic q-Markov semigroups and speed of convergence of $q$-algorithms, Infin. Dimens. Anal. Quantum Probab. Relat. Top. 9 (2006) 567-594.

2. L. Accardi, F. Fagnola and R. Quezada, On three new principles in nonequilibrium statistical mechanics and Markov generators of weak coupling limit type, Infin. Dimens. Anal. Quantum Probab. Relat. Top. 19 (2016) 1650009, doi:10.1142/S0219025716500090.

3. L. Accardi and S. Kozyrev, Lectures on quantum interacting particle systems, in Quantum Interacting Particle Systems, $Q P-P Q$, eds. L. Accardi and F. Fagnola, Vol. XIV (World Scientific, 2002), pp. 1-195.

4. B. V. Rajarama Bhat, F. Fagnola and M. Skeide, Maximal commutative subalgebras invariant for CP-maps: (counter-)examples, Infin. Dimens. Anal. Quantum Probab. Relat. Top. 11 (2008) 523-539.

5. J. R. Bolaños-Servin and R. Carbone, Spectral properties of circulant quantum markov semigroups, Open Syst. Inf. Dyn. 21 (2014) 1450007.

6. J. R. Bolaños-Servin and R. Quezada, A cycle decomposition and entropy production for circulant quantum Markov semigroups, Infin. Dimens. Anal. Quantum Probab. Relat. Top. 16 (2013) 1350016, doi:10.1142/S0219025713500161.

7. R. Carbone and F. Fagnola, Exponential $L^{2}$-convergence of quantum Markov semigroups on $\mathcal{B}(h)$, Mat. Zametki 68 (2000) 523-538 [translation in Math. Notes 68 (2000) 452-463].

8. R. Carbone, E. Sasso and V. Umanità, Structure of generic quantum Markov semigroups, Infin. Dimens. Anal. Quantum Probab. Relat. Top. 20 (2017) 1750012.

9. F. Fagnola, Quantum Markov semigroups and quantum Markov flows, Proyecciones 18 (1999) 1-144.

10. K. R. Parthasarathy, An Introduction to Quantum Stochastic Calculus (Springer Basel AG, 1992). 